\title{
ORIENTATION BIBLIOGRAPHIQUE
}

AFTALION Fred, Histoire de la chimie, Masson, Paris, 1997.

BAILLOT Rémi, Georges Claude, le génie fourvoyé. Créateur d'Air Liquide, du tube au néon, de l'énergie thermique des mers, EDP Sciences, Paris, 2010.

BAUD Paul, L'industrie chimique en France. Étude historique et géographique, Masson et $\mathrm{C}^{\mathrm{ie}}$, Paris, 1932.

BAUD Paul, La grande industrie chimique et ses récents progrès, GauthierVillars, Paris, 1937.

BAUD Paul, Traité de chimie industrielle. T. 1. Grande Industrie chimique, Masson et Cie, Paris, 1951 (4e édition).

BENOIT Serge et EMPTOZ Gérard, "Les collections de chimie de la Société d'encouragement pour l'industrie nationale », Revue d'histoire des sciences, 69/1, 137-151 (janvier-juin 2016).

BENOIT Serge, EMPTOZ Gérard et WORONOFF Denis (éd.), Encourager l'innovation en France et en Europe, Éd. CTHS, Paris, 2006.

BLANCHARD Raoul. "La grande industrie chimique dans la France du Sud-Est », Revue de géographie alpine, 16/3 (1928), 561-624.

CARON François, Histoire économique de la France, XIX $-X X^{e}$ siècles, Armand Colin, Paris, 1981.

CHAPLET Auguste, Où en est la chimie industrielle? Gauthier-Villars, Paris, 1928.

CHARDONNET Jean, «L'industrie chimique des sous-produits de la houille en France à la veille de la guerre », Annales de géographie, 54/295 (1945), 180-191.

CHAUVEAU Sophie, L'Invention pharmaceutique. La pharmacie française entre l'État et la société au $X X^{e}$ siècle, Institut d'édition Sanofi-Synthélabo, Paris, 1999.

CLARKE Jackie, France in the age of organization: Factory, home and nation from the 1920s to Vichy, Berghahn Books, New York, 2011. 
DAUMALIN Xavier, «Désindustrialisation et ré-industrialisation à Marseille fin $\mathrm{XIX}^{\mathrm{e}}$ - début $\mathrm{XX}^{\mathrm{e}}$ siècle », Rives méditerranéennes, 46/3 (2014), 47-62.

DAUMALIN Xavier, Du sel au pétrole : l'industrie chimique de Marseille, Paul Tacussel, Marseille, 2003.

DAVIET Jean-Pierre, "L'industrie chimique française au tournant de la seconde industrialisation (1860-1939) », Culture technique, 23 (1991), 5367.

DAVIET Jean-Pierre, Un destin international : La Compagnie de SaintGobain de 1830 à 1939, Éditions des archives contemporaines, Paris, 1988.

DI MEO Guy, «L'industrie française de la parfumerie », Annales de Géographie, 82/452, 454-476 (1973).

DUMAS Maurice, «La politique d'orientation de la recherche scientifique et technique : Le progrès dans l'industrie chimique, 1919-1939 », Cahiers de l'Institut de science économique appliquée (mars 1962), p. 33-49.

DUPONT Georges, Essences de térébenthine, Masson et Gauthier-Villars, Bordeaux, 1926.

EMPTOZ Gérard, «La nouvelle chimie de l'azote dans l'entre-deuxguerres ", L'Actualité chimique, 381, 40-46 (janvier 2014).

EMPTOZ Gérard, "La création de l'Air Liquide au début du $\mathrm{XX}^{\mathrm{e}}$ siècle », in Marseille Jacques (dir.), Créateurs et créations d'entreprises de la Révolution industrielle à nos jours, ADHE, Paris, 2000, p. 677-692.

FAUQUE Danielle, « La documentation au cœur de la réorganisation de la chimie dans l'entre-deux-guerres : Rôle des sociétés savantes et institutions françaises dans le contexte international », Revue d'histoire des sciences, 69/1, 41-75 (janvier-juin 2016).

FAUQUE Danielle et EMPTOZ Gérard, «Les chimistes, leurs institutions et leurs sociétés savantes entre les deux guerres. Introduction », L'Actualité chimique, 378-379, 120-123 (octobre-novembre 2013).

FAUQUE Danielle, «French chemists and the international reorganisation of chemistry after World War I », Ambix, 58/2, 116-135 (July 2011).

FAUQUE Danielle, " Les congrès de chimie industrielle dans l'entre-deuxguerres : vitrine des relations entre l'industrie, la science et la politique », L'Actualité chimique, 380, 39-44 (décembre 2013).

FAUQUE Danielle (dir.), «Réorganiser la chimie entre les deux guerres : Rôle des sociétés savantes et institutions françaises dans le contexte national et international », Revue d'histoire des sciences, 69/1 (janvier-juin 2016), dossier thématique, 5-151. 
FELL Ulrike (dir.), Chimie et industrie en Europe. L'apport des sociétés savantes industrielles du XIX $X^{e}$ siècle à nos jours, Éditions des archives contemporaines, Paris, 2001.

FELL Ulrike, "Quelle liaison entre science et industrie ? La Société de chimie industrielle entre les deux guerres, 1917-1939 », in Fell U. (dir.), Chimie et industrie en Europe, op.cit., p. 69-95.

GALVEZ-BEHAR Gabriel, "The "French connection": French scientists and international debates on scientific property during the Interwar Period », (2013) : https://halshs.archives-ouvertes.fr/halshs-00839580

GÉRARD, Jean, 1914-1924. Dix ans d'efforts scientifiques, industriels et coloniaux, Chimie et Industrie, Paris, 1926 (2t).

GRELON André, «Formation et carrière des ingénieurs en France (18801939) », in Bergeron Louis et Bourdelais Patrice (dir.), La France n'est-elle pas douée pour l'industrie?, Belin, Paris, 1998, p. 231-274.

GRELON André (dir.), Les ingénieurs de la crise. Titre et profession entre les deux guerres, Paris, Éditions de l'EHESS, 1986.

GROSSETTI Michel, DETREZ Claude, "Science d'ingénieurs et Sciences pour l'ingénieur : l'exemple du génie chimique », 1999, disponible sur https://halshs.archives-ouvertes.fr/halshs-00476385 (http://www.rechercheisidore.fr/search/resource/?uri=10670/1.59yjmf)

HABER Ludwig Fritz, The chemical industry: 1900-1930, international growth and technological change, Oxford University Press, Oxford, 1971.

HOUDRY Eugène, "Historique du cracking catalytique dans l'industrie du pétrole », Bulletin de l'Association française des techniciens du pétrole (1956), p. 203-212.

JOLY Hervé, ROBERT François (avec GIANDOU Alexandre), Entreprises et pouvoir économique dans la région Rhône-Alpes (1920-1954), Centre Pierre Léon d'histoire économique et sociale, Lyon, 2003.

JOLY Hervé, Les Gillet de Lyon. Fortunes d'une grande famille industrielle (1838-2015), Droz, Genève, 2015.

LAFERRÈRE Michel, «Esquisse d'une géographie des industries chimiques dans la région lyonnaise », Revue de géographie de Lyon, 27/3, 303-308 (1952).

LAFERRÈRE Michel, «Les industries chimiques de la région lyonnaise », Les Études rhodaniennes, 27/3, 219-256 (1952).

LAFERRÈRE Michel, Lyon, ville industrielle : essai d'une géographie urbaine des techniques et des entreprises, Presses universitaires de France, Paris, 1960. 
LAMARD Pierre et STOSKOPF Nicolas (dir.), L'industrie chimique en question, Éd. Picard, Paris, 2010.

LANGLINAY Erik, "Kuhlmann at War (1914-1924)» in MacLeod Roy, Johnson Jeffrey A. (ed.), Frontline and Factory, Comparative Perspectives on the Chemical Industry at War, 1914-1924, Springer, Dordrecht, 2006, p. 145-166.

LÉGER Jean-Étienne, Une grande entreprise dans la chimie française : Kuhlmann, 1825-1982. Nouvelles Éditions Debresse, Paris, 1988.

LESTEL Laurence (coord.), Itinéraires de chimistes (1857-2007), 150 ans de chimie en France avec les présidents de la SFC, EDP Sciences, Paris, 2007.

LETTÉ Michel, «Chimie, chimistes et rationalisation sous les auspices du ministre du Commerce et de l'Industrie Étienne Clémentel (1917-1919)», Revue d'histoire des sciences, 69/1, 19-40 (janvier-juin 2016).

LETTÉ Michel, «Le rapport d'Étienne Clémentel (1919): L’avènement administratif des technocrates et de la rationalisation », Documents pour l'histoire des techniques, 20, 167-181 (décembre 2011).

LUCAS J.-H., « Les engrais chimiques en 1925 », L'industrie chimique, 151 352-354 (1926).

MACKIE Robin et ROBERTS Gerrylynn K., "Un secteur à part ? Les chimistes industriels et la Society of Chemical Industry dans le contexte de la communauté chimique britannique ", in Fell U. (dir), Chimie et industrie en Europe, op. cit., p. 127-147, (trad. Nathalie Jas).

MOUREU Charles, La chimie et la guerre, science et avenir, Masson, Paris, 1920.

MOURIAUX René, «Le syndicalisme des ingénieurs et cadres. Histoire et historiographies », Culture technique, 12 (mars 1984). $\mathrm{N}^{\circ}$ spécial «Les ingénieurs ».

NYE Mary Jo, Science in the Provinces: Scientific communities and provincial leadership in France, 1860-1930, University of California Press, Berkeley, 1986.

PACAUT Marcel, «L'industrie des produits pharmaceutiques à Lyon », Les Études rhodaniennes, 21/1-2, 55-66 (1946).

PAUL Harry W., From knowledge to power: The rise of the science empire in France, 1860-1939, Cambridge University Press, Cambridge, 1985.

SAKUDO Jun, Les entreprises de la chimie en France de 1860 à 1932, P.I.E. Peter Lang, Bruxelles, 2011. 
SAUVY Alfred, Histoire économique de la France entre les deux guerres. De l'armistice à la dévaluation de la Livre, Fayard, Paris, 1965 (3 vols).

THORBURN Burns D., DEELSTRA Hendrik, "The origins and impact of the international congresses of applied chemistry, 1894-1912», Microchim Acta, 172, 277-283 (2011).

THORBURN Burns D., DEELSTRA H., « Establishing a vital tradition: the series of international congresses of applied chemistry, 1894-1912», Chemistry International, 33/4, 11-14 (2011).

TRAVIS Anthony S., SCHRÖTER Harm G., Homburg Ernst, Morris Peter J.T (eds.), Determinants in the evolution of the european chemical industry, 1900-1939: New technologies, political frameworks, markets and companies, Kluwer Academic Publishers, Dordrecht, 1998. 
